Case Report

\title{
Case Report of a Laparoscopic Primary Diaphragmatic Repair in Acute Penetrating Trauma: Is It Within the Realm of an Acute Care Surgeon?
}

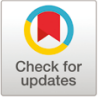

\author{
Yagan Pillay ${ }^{a, *}$, Cheyenne Vetter ${ }^{b}$ \\ ${ }^{a}$ Department of General Surgery, University of Saskatchewan, Saskatchewan, Canada \\ ${ }^{b}$ Family Medicine Surgery, Alberta Health Services, Wainwright, Alberta, Canada
}

Article history:

Received: August 16, 2020

Revised: October 16, 2020

Accepted: November 9, 2020

\section{${ }^{*}$ Corresponding Author:}

Yagan Pillay

Department of General Surgery, University of

Saskatchewan, Health Sciences Building,

107 Wiggins Rd. B419, Saskatoon, S7N

oW8, Saskatchewan, Canada

Email: yagan2pillay@yahoo.ca

\section{ORCID}

Yagan Pillay

https://orcid.org/0000-0003-1254-3179

Cheyenne Vetter

https://orcid.org/0000-0002-9431-8305

\begin{abstract}
The laparoscopic repair of traumatic diaphragmatic injuries in the acute phase has not been well established. The current consensus view is an elective repair by a surgeon with an advanced laparoscopic skill set. This case report illustrates the position that with judicious patient selection an acute repair is not only possible but well within the realm of an acute care surgeon. Anterior diaphragmatic injuries with penetrating trauma can even be repaired primarily without the need for prosthetic reinforcement. It is hoped that this case report will add to the surgical armamentarium of the acute care surgeon.
\end{abstract}

Keywords: diaphragm injury, laparoscopic repair, penetrating diaphragmatic trauma, acute care surgery

\section{Introduction}

Traumatic diaphragmatic injury (TDI) makes up a miniscule component of all trauma patients with a reported incidence of $0.46 \%$ in the published literature [1]. Two thirds of all cases are due to penetrating trauma with the majority on the left hemidiaphragm and usually involves the anterior portion of the diaphragm [2]. We present a case report of a left sided TDI, its diagnosis and laparoscopic repair in the acute setting which is not the recognised norm for this type of injury. This can be undertaken through judicious patient selection and the requisite laparoscopic surgical skillset.

\section{Case Report}

A twenty three year-old female presented to the emergency room with a stab injury to her left thoraco-abdomen. She was hemodynamically stable and her primary trauma survey revealed a left sided pneumothorax on erect chest X-ray (CXR).

Her pneumothorax was treated by the emergency room physician with a left sided intercostal drain.

Her repeat CXR (Figure 1A) to confirm drain position showed free air under the right hemidiaphragm and she was referred to the acute care surgery team.

Her abdominal examination on the secondary trauma survey was equivocal with no clinically palpable masses, rebound tenderness or involuntary guarding. She would not volunteer information on the aetiology of her penetrating trauma. She remained hemodynamically stable throughout her admission and displayed no signs of respiratory compromise.

A computerised tomography (CT) scan (Figures 1B-D) of her abdomen showed anterior laceration of her left hemidiaphragm with omental evisceration into the pleural space. She had no solid or hollow visceral injuries on radiological imaging and no free fluid in the peritoneal or pleural cavities. Her 
hematological parameters showed a leucocytosis but an expanded chemistry profile was normal.

A urinary drug screen was negative.

The patient was admitted to the surgery floor for observation after she initially refused a diagnostic laparoscopy at presentation to the emergency department.

Upon further discussion two hours later, she agreed to surgery and signed an informed consent for a diagnostic laparoscopy as well as video recording of the surgery.
Intra-operatively the diaphragmatic laceration was noted and the viable incarcerated omentum (Figures 2A-D) reduced into the peritoneal cavity. Visualisation of the left pleural space through the diaphragmatic laceration showed the intercostal drain in situ with no obvious lung injury (Figure 2E).

The anterior laceration was repaired with $2-0 \mathrm{~V}-\operatorname{loc}^{\circ}$ non absorbable continuous suture (Figure $3 \mathrm{~A}$ ) and the suture was secured to the diaphragm using gallbladder clips (Figures 3B and C).
(A)

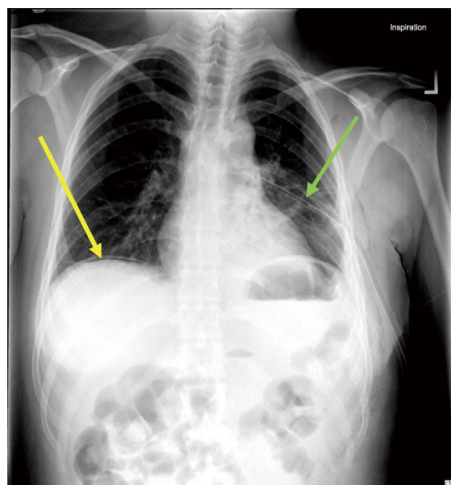

(B)

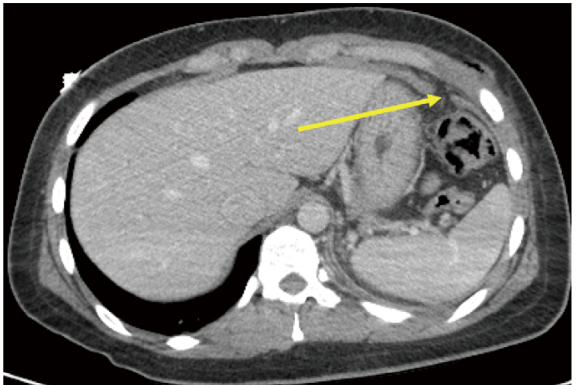

Figure 1. (A) Erect CXR showing free air under the right hemidiaphragm (yellow arrow) with a left intercostal drain in situ (green arrow). (B) CT scan axial view showing the left diaphragmatic laceration (yellow arrow). (C) CT scan coronal view showing the diaphragmatic laceration (yellow arrow). (D) CT scan sagittal view showing an anterior diaphragmatic laceration (yellow arrow).
(C)



(D)



(A)

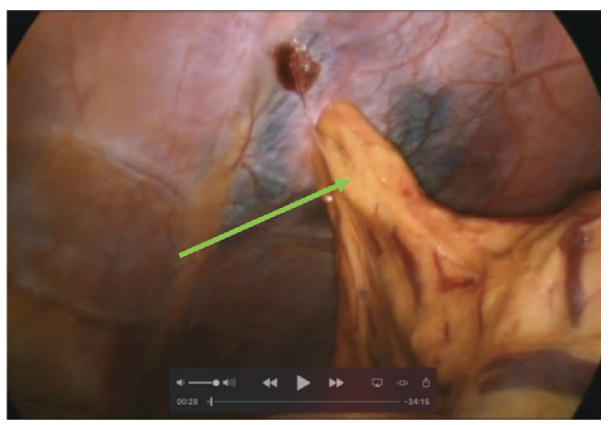

(D)

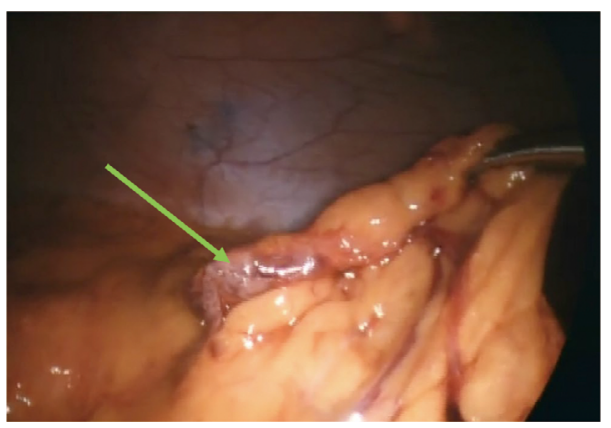

(B)

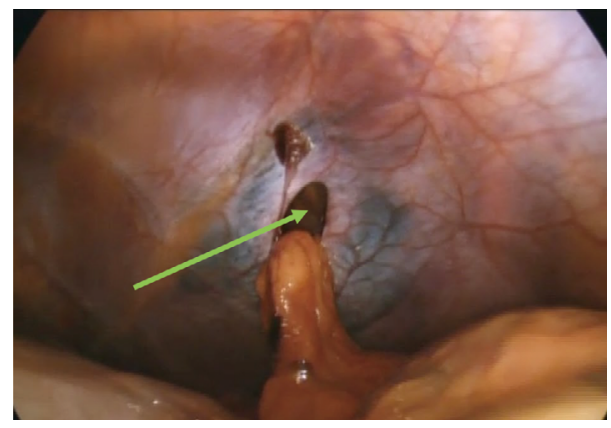

(E)



(C)

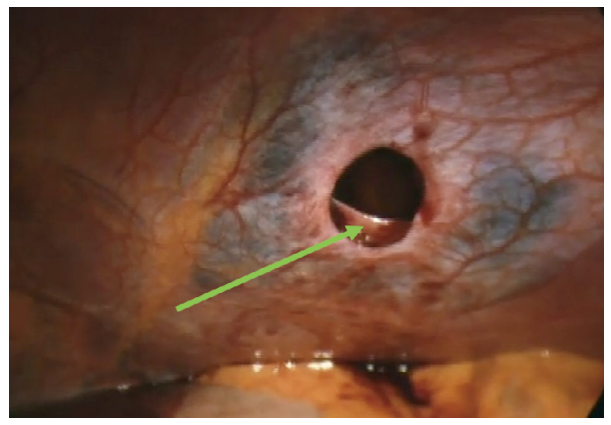

Figure 2. (A) Laparoscopic view of the diaphragmatic laceration with incarcerated omental contents (green arrow). (B) Partial omental reduction through pneumoperitoneum (green arrow). (C) Diaphragmatic laceration with the pleura visible (green arrow). (D) Viable incarcerated omentum (green arrow). (E) Thoracoscopic view through the laceration showing the anterior ribs (yellow arrow), Left lung (green arrow) and intercostal drain in situ (red arrow). 
(A)

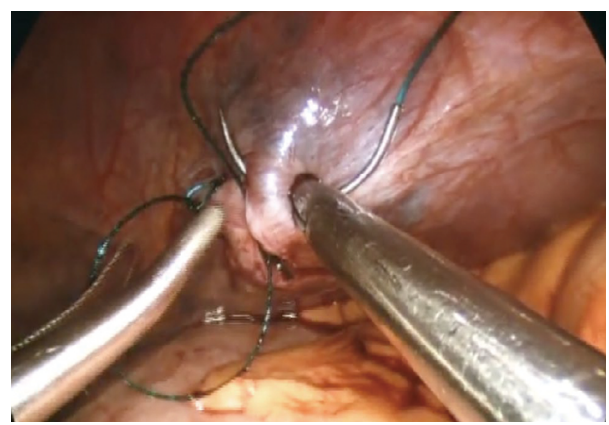

(B)



(C)

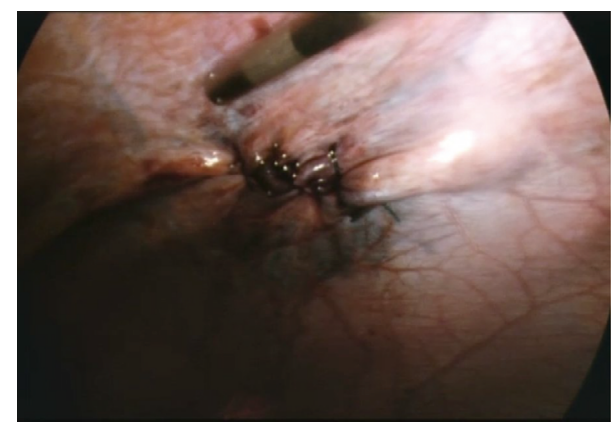

Figure 3. (A) Laparoscopic suturing of the laceration with 2-0 V-Loc ${ }^{\circ}$ non absorbable suture. (B) Suture stabilisation with gallbladder clips (yellow arrow). (C) The completed left sided anterior diaphragmatic repair.

Laparoscopic evaluation of the right and left upper quadrants of the abdominal cavity did not reveal any concomitant injury to the spleen, stomach, oesophagus or liver.

Her post-operative recovery was uneventful and the intercostal drain was removed after 24 hours. She was discharged home on the second post-operative day.

She has been lost to surgical follow up and did not attend her ambulatory care appointment two weeks later. All attempts to contact her have proven unsuccessful.

\section{Discussion}

Penetrating traumatic diaphragmatic injury (TDI) in the acute phase has not been clearly dealt with, in the English literature. In a stable patient with no obvious clinical signs of peritonism, the different radiological modalities such as CT scan or MRI are not very accurate in diagnosing a diaphragmatic injury. The sensitivity of a CT scan approaches $80 \%$ in the absence of concomitant abdominal visceral injuries $[2,3]$.

The routine use of diagnostic laparoscopy has been recommended to eliminate diagnostic doubt with a sensitivity approaching $100 \%$ [4]. Laparoscopy is thought to prevent the complication of diaphragmatic herniation of abdominal viscera which would make for a difficult surgical repair at a later date [5]. Hernial visceral strangulation while rare, can have devastating consequences with a mortality rate approaching $20 \%[5]$.

The acute repair of the diaphragm is controversial for two reasons. One is the potential contamination of the field, with perforated bowel content which precludes the use of a nonabsorbable mesh in a wide defect or a posterior defect. The use of biological mesh in a contaminated repair is not well described. The second is that the requisite surgical skill set is often an advanced laparoscopic skill. The current standard of care in a contaminated field as per the East trauma guidelines is an open repair [1].
Posterior diaphragmatic defects are difficult to close primarily and often require a non-absorbable mesh reinforcement [6]. This has been postulated to be more efficient if undertaken on an elective basis rather than in the acute setting, by an experienced surgeon with an advanced laparoscopic skill set [7].

This report illustrates that this repair is feasible in the acute phase and well within the surgical realm of an acute care surgeon. The ability to select the specific type of injury and the exclusion of concomitant injuries allows for an effective acute repair. Anterior diaphragmatic injuries are more amenable to a primary repair and the goal remains a tension free repair [8]. The majority of penetrating trauma injuries are anterior diaphragmatic injuries.

A minimally invasive approach shortens the recovery time and minimizes operative complications. The surgical repair in the acute phase also avoids a second surgery at a later date and the cost implications of the elective repair should also be taken into account.

We were unable to contact the patient who has unfortunately been lost to surgical follow up and continued surveillance of the diaphragmatic repair has not been possible.

\section{Conclusion}

We present a case report of an acute penetrating diaphragmatic laceration which was laparoscopically repaired in the acute phase through appropriate patient selection and shown to be within the realm of the laparoscopic surgical skill set of an acute care surgeon.

\section{Conflicts of Interest}

The authors have no conflicts of interest to declare. 


\section{References}

[1] McDonald AA, Robinson BRH, Alarcon L, Bosarge PL, Dorion H, Haut ER, et al. Evaluation and management of traumatic diaphragmatic injuries: A Practice Management Guideline from the Eastern Association for the Surgery of Trauma. J Trauma Acute Care Surg 2018;85(1):198-207.

[2] Lim BL, Li TT, Ming TC, Marxengel LA, Eillyne S. Traumatic diaphragmatic injuries: a retrospective review of a 12-year experience at a tertiary trauma centre. Singapore Med J 2017;58(10):595-600.

[3] Bala M, Faroja M. Laparoscopic Repair of Acute Traumatic Diaphragmatic Hernia: Is Proximity to Esophageal Hiatus a Contraindication? Austin J Trauma Treat 2015;2(1):1005.
[4] O'Malley E, Boyle E, O'Callaghan A, Coffey JC, Walsh SR. Role of laparoscopy in penetrating abdominal trauma: a systematic review. World J Surg 2013;37(1):113-22.

[5] Nguyen P, Davis B, Tran DD. Laparoscopic Repair of Diaphragmatic Rupture: A Case Report with Radiological and Surgical Correlation. Case Rep Surg 2017;2017:4159108.

[6] Xenaki S, Lasithiotakis K, Andreou A, Chrysos E, Chalkiadakis G. Laparoscopic repair of posttraumatic diaphragmatic rupture. Report of three cases. Int J Surg Case Rep 2014;5(9):601-4.

[7] Bhatti UH, Dawani S. Large bowel obstruction complicating a posttraumatic diaphragmatic hernia. Singapore Med J 2015;56(4):e56-8.

[8] Mohamed M, Al-Hillan A, Shah J, Zurkovsky E, Asif A, Hossain M. Symptomatic congenital Morgagni hernia presenting as a chest pain: a case report. J Med Case Rep 2020;14(1):13. 